\title{
A DELAY-LINE POSITION READOUT FOR A HEAVY-ION PROPORTIONAL COUNTER
}

\author{
C.P.M. VAN ENGELEN, R.O. BLAAUBOER and R. KAMERMANS \\ Fysisch Laboratorium, Rijksuniversiteit Utrecht, P.O. Box 80.000, 3508 TA Utrecht, The Netherlands
}

Received 5 September 1983

A delay-line position readout system connected to the proportional counter of a gas-filled heavy-ion detector was constructed and tested in a magnetic spectrograph. The measured position resolution was less than $0.3 \mathrm{~mm}$ (fwhm) which is $0.1 \%$ of the full detector length $(250 \mathrm{~mm})$. The differential nonlinearity of the readout system was found to be less than $1.0 \mathrm{~mm}$.

\section{Introduction}

In the last decade gas-filled proportional counters have been used successfully as focal-plane detectors in magnetic spectrographs [1]. For the detection of light-heavy ions a combination of an ionization chamber and proportional counters was developed both in Rochester [2] and in Argonne [3] for use in an Enge split-pole spectrograph. In these detectors two proportional counters with resistive-wire position readout are incorporated in the gas volume of a $\Delta E / E$ ionization chamber.

Although the performance of the resistive-wire readout is quite good with a position resolution better than $0.7 \mathrm{~mm}$ (fwhm) for a total length of the wire of $250 \mathrm{~mm}$ [2], there are some disadvantages: (1) the gain-matching procedure required in order to obtain a good linearity and position resolution is time consuming, (2) if the detector is filled with isobutane, regularly used for its high stopping power, the resistive wire will be polluted by products of cracking, and (3) the resistive wire (12 $\mu \mathrm{m} \mathrm{NiCr}$ ) is quite fragile and may contain inhomogeneities.

An alternative approach is to install a delay-line readout system [4]. Then no gain-matching procedure is required and, since the delay line will be coupled to the cathode of a proportional counter, pollution of the anode wire will not affect the position resolution and linearity. Moreover the fragile $\mathrm{NiCr}$ wire can be replaced by one or more stronger tungsten wires. The position resolution that can be obtained with a delay-line readout is good, for lightly ionizing particles a resolution of $0.25 \mathrm{~mm}$ ( $\mathrm{fwhm}$ ) for a line of $250 \mathrm{~mm}$ length has been observed [5].

The Enge split-pole spectrograph of our laboratory is equipped with a gas-filled detector of the "Rochester" type [2]. In this article we report on the results of a delay-line readout system developed for this type of detector. The delay line and the associated electronics are described in section 2 , as well as a small gas-filled detector which was constructed in order to test the delay-line readout system. The resolution and linearity of the readout system were measured under beam conditions by placing the test detector in the split-pole spectrograph. The results of these experiments are presented and discussed in section 3 and the conclusions can be found in section 4 .

\section{Experimental setup}

\subsection{Delay line and electronics}

The delay line was of a discrete type [6] with 100 taps at a mutual distance of 0.1 inch. The 9 ns delay per tap results in a total delay time of $0.9 \mu \mathrm{s}$. With a characteristic impedance of $800 \Omega$ and a dc resistance of $170 \Omega$ the attenuation coefficient of this line is 0.1 . We have also used a continuous delay line [7], however, the discrete line has some advantages: (1) the rigidity of the line, (2) the absence of forerunners in front of the signal and (3) the charge induced on the cathode of the proportional counter is directly put into the line, contrary to the continuous line where the charge has to be coupled capacitively into the line.

The electronic resolution and linearity of the line were tested with the setup given in fig. 1. Standard charge-sensitive preamplifiers (Canberra 2003) were installed with two $800 \Omega$ resistors placed in series at the two ends of the delay line in order to match the impedance of the delay line. The signals from the preamplifiers were processed by timing filter amplifiers (Ortec $474, t_{\text {diff }}=200 \mathrm{~ns}, t_{\text {int }}=100 \mathrm{ns)}$ and fed into constant fraction discriminators (Ortec 583, $f=0.2$, delay 120 ns). The whole line was scanned with a pulser, which was connected to the line via a charge terminator. The risetime of the pulses from the delay line varied between 


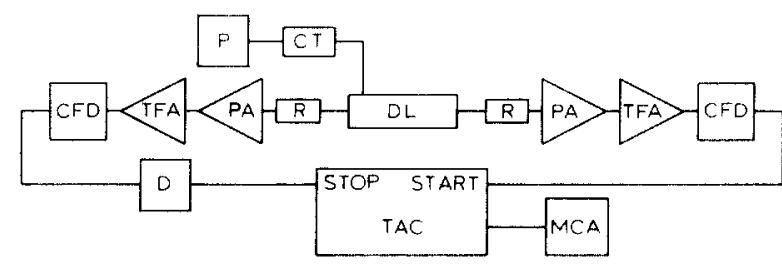

Fig. 1. Electronics for the delay-line readout: P: pulser; CT: charge terminator; DL: delay line; $\mathrm{R}: 800 \Omega$ resistor to match the impedance of the delay line; PA: preamplifier (Canberra 2003); TFA: timing filter amplifier (Ortec 474, $t_{\text {diff }}=200 \mathrm{ns,}$ $t_{\text {int }}=100 \mathrm{~ns}$ ); CFD: constant fraction discriminator (Ortec 583, $t_{\text {delay }}=120 \mathrm{~ns}$ ); D: delay $0.95 \mu \mathrm{s}$; TAC: time to amplitude converter (Canberra 2043, mage $2 \mu \mathrm{s}$ ); MCA: multichannel analyzer.

$50 \mathrm{~ns}$ for a pulse with no delay (the risetime of the input pulse) and $150 \mathrm{~ns}$ for a pulse with a full delay of $0.9 \mu \mathrm{s}$. Using a linear fit the differential nonlinearity was less than $0.5 \mathrm{~mm}$ in the middle of the line, increasing to 1.0 $\mathrm{mm}$ at both ends. Using a quadratic fit the difference between the fit and the data was less than $0.3 \mathrm{~mm}$ over the full length of the line. For a preamplifier pulse with an amplitude of $100 \mathrm{mV}$, which is a typical value for the height of a pulse from the cathode of a proportional counter, the electronic resolution (fwhm) was found to be $0.2 \mathrm{~mm}$.

\subsection{The test detector}

The delay-line readout system was tested in a small gas-filled detector which is shown in fig. 2. The ionization part simply consists of a cathode and a grounded anode, which create a vertical electrical field similar to the one in the "Rochester" type detector. By applying a bias to the aluminized entrance window the field disturbance due to the entrance window could be minimized. Since we were not interested in the energy signals of the anode, the Frish grid could be omitted. The proportional counter was placed above the anode of the ionization chamber. This anode partly consists of a small grid in order to make it transparant for electrons drifting upwards into the proportional counter. The cathode of the proportional counter was constructed from three parts of printed circuit board, on which 100 stripes with a width of $2 \mathrm{~mm}$ at intervals of $2.54 \mathrm{~mm}$ $(0.1$ inch) were etched. The corresponding stripes on the three parts were electrically connected to each other and to a tap of the delay line. For the anode of the proportional counter a $\mathrm{NiCr}$ resistive wire (resistance over 250 $\mathrm{mm}: 2.5 \mathrm{k} \Omega$, diameter $12 \mu \mathrm{m}$ ) was installed. In this way it was possible to directly compare the resistive-wire readout with the delay-line readout. Two charge-sensitive preamplifiers (Canberra 2003) followed by standard main amplifiers (Ortec 452, shaping time $0.5 \mu \mathrm{s}$ ) were a

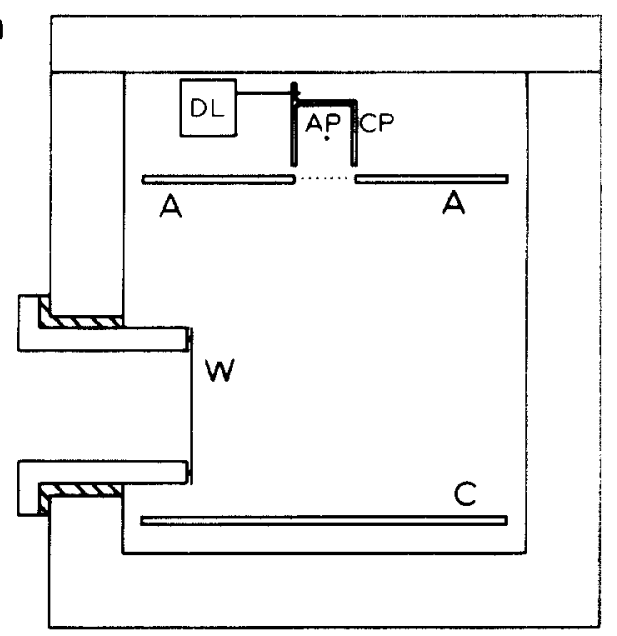

b

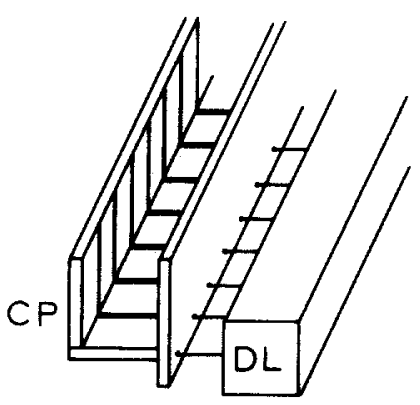

Fig. 2. (a) Side view of the test detector: $W$ : window (aluminized mylar, $2 \mu \mathrm{m}$ ); $\mathrm{C}$ : cathode; $\mathrm{A}$ : anode; $\mathrm{CP}$ : cathode of the proportional counter; AP: anode of the proportional counter ( $\mathrm{NiCr}$ wire, diameter $12 \mu \mathrm{m}$, total resistance $2.5 \mathrm{k} \Omega$ ); DL: delay line. (b) Perspective view of the striped cathode of the proportional counter (CP) connected to the delay line (DL).

used for the charge-division readout from the resistive anode.

\section{Experimental results}

\subsection{Electron charge distribution}

Two conditions have to be fulfilled in order to achieve a position resolution better than the stripe width $(2.5 \mathrm{~mm})$ : (1) the charge must be induced on at least three stripes to reduce systematic errors [8] and (2) the risetime of the pulse must be longer than the delay between three stripes, since in that case the pulse represents the mean position of the avalanche around the anode. The second condition is easily fulfilled for a 
delay per tap of $9 \mathrm{~ns}$ and an integration time of the timing filter amplifier of $100 \mathrm{~ns}$. By measuring the distribution of the induced charge along the cathode the first condition could be tested. In this experiment one stripe of the cathode was connected to a charge-sensitive preamplifier with the delay line removed while all other stripes were grounded. A collimated ${ }^{241} \mathrm{Am} \alpha$ source was moved along the detector, which was filled with isobutane at a pressure of 50 Torr. For several positions the amount of charge induced on the stripe was measured, the results are shown in fig. 3. This measurement was repeated for several voltages on the anode wire $(900,1000$ and $1050 \mathrm{~V}$, respectively), however, it was found that the shape of the charge distribution along the cathode remained the same.

The charge pulse on the cathode is due to electrons created in an avalanche only a few wire diameters away from the anode wire. Therefore the shape of the charge distribution $(Q)$ can in a first approximation be described with the assumption of a point charge which is located between two parallel planes [8]:

$Q(x)=\frac{C}{\cosh (\pi x / 2 D)}$

Here $D$ is the distance between the point charge and both cathode planes, $x$ is the distance along the cathode planes between the projection of the point charge on the planes and the position where the charge is measured and $C$ is a scaling factor. The solid line in fig. 3 corresponds to this function with $D=4.7 \mathrm{~mm}$, in good agreement with the actual distance of about $5 \mathrm{~mm}$ between the anode and the rectangular cathode.

With this function it is possible to calculate the systematic error in the position determination, assuming that the output pulse consists of the pulses from 10 stripes around the maximum. In the middle of the line

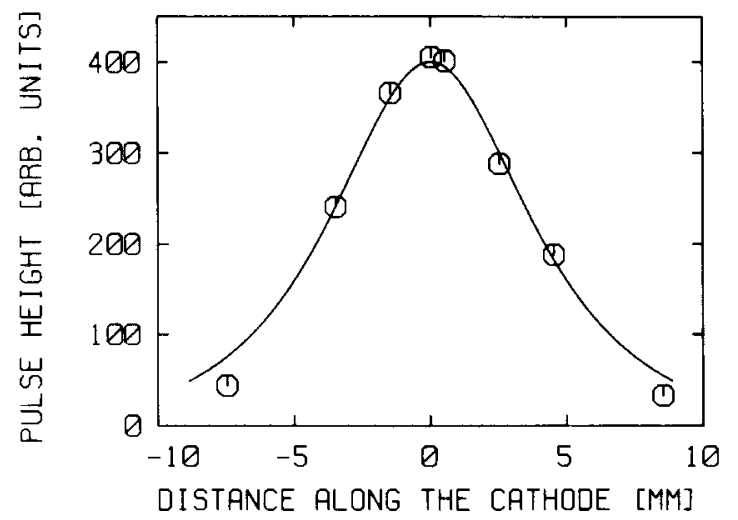

Fig. 3. The charge induced on a cathode stripe as a function of the distance along the cathode between the avalanche and the stripe. The solid line is a fit to the data which is described in the text. this systematic error is only $0.1 \mathrm{~mm}$, small compared to the electronic resolution of $0.2 \mathrm{~mm}$ (section 2.1). At both ends of the line the systematic error amounts to 3.3 $\mathrm{mm}$, due to the fact that only half of the charge distribution is induced on the cathode stripes. This edge effect becomes negligible already at $10 \mathrm{~mm}$ from both ends.

\subsection{Position resolution and linearity}

The linearity and resolution of the position readout systems under beam conditions were determined by placing the test detector in an Enge split-pole spectrograph. The proportional counter was positioned perpendicular to the path of the ions as is the case for the "Rochester" type detector. A ${ }^{12} \mathrm{C}$ beam with an energy of $36 \mathrm{MeV}$ accelerated by the EN tandem of the Van de Graaff Laboratory in Utrecht was elastically scattered from a $100 \mu \mathrm{g} / \mathrm{cm}^{2}$ thick Au target. The spectrograph was placed at a scattering angle of $20^{\circ}$. The horizontal opening angle was taken quite small $\left(0.3^{\circ}\right)$ in order to minimize the divergency of the ion paths in the spectrograph. By changing the magnetic field of the spectrograph the performance of the detector could be tested over its whole range. Isobutane at a pressure of 50 Torr was used as a counting gas, which resulted in an energy loss under the proportional counter of $600 \mathrm{keV}$. The cathode of the ionization chamber was at $-400 \mathrm{~V}$, its anode grounded, whereas the anode of the proportional counter was operated at a bias of $900 \mathrm{~V}$. The electronics setup for the delay-line readout was identical to the one shown in fig. 2 except for the TAC which was replaced by a TDC (LeCroy 2228A, range $2 \mu \mathrm{s}$ ). In this setup the height of the pulse after the preamplifier varied between 100 and $300 \mathrm{mV}$.

First the interpolation capability of the delay-line readout was measured. The magnetic field in the spectrograph was varied in small steps in order to shift the ion paths in the detector in steps of $0.33 \mathrm{~mm}$. Data were taken for fifteen positions covering a length of $50 \mathrm{~mm}$ (two stripes) in the centre of the detector. Linear fits to the data points from the delay-line and charge-division readout were made and the differences between a fit and the data (the residues) give the differential nonlinearity, which is shown in fig. 4. The maximum nonlinearity amounts to $80 \mu \mathrm{m}$ for the resistive-wire and 60 $\mu \mathrm{m}$ for the delay-line readout. The similar behaviour of the residues for the delay-line and charge-division readout indicates a variation of step width due to the accuracy in the determination of the magnetic field strength rather than nonlinearities in the individual readout systems. No indication for a $2.5 \mathrm{~mm}$ discontinuity in the data from the delay-line readout can be observed, demonstrating the interpolation effect discussed in the previous section.

Secondly the linearity and position resolution were 


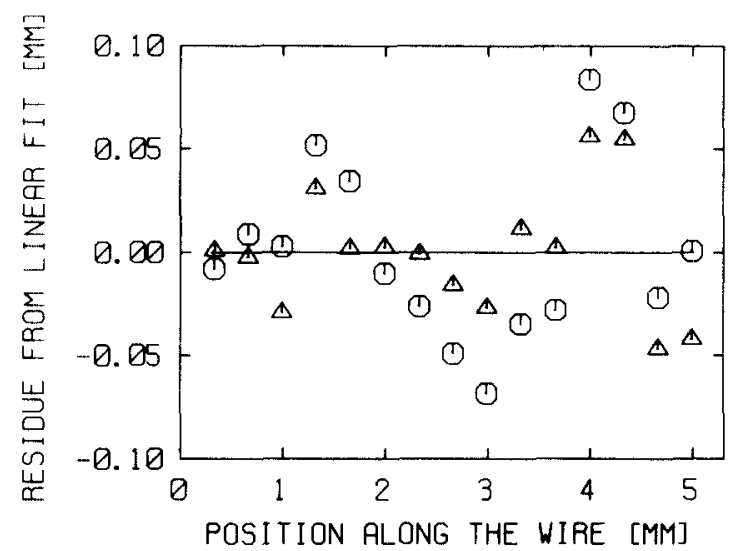

Fig. 4. The difference between the measured positions and a linear fit to the data for 15 positions in the centre of the counter. The $5 \mathrm{~mm}$ cover two stripes of the cathode. The triangles give the residues for the delay-line readout, the circles for the charge-division readout.

measured over the full length of the counter by placing a mask in front of the detector. This mask consisted of 13 vertical slits (width $0.2 \mathrm{~mm}$ ) at mutual distances of $20 \mathrm{~mm}$. Data were taken for ions entering the detector through all 13 slits by shifting the ${ }^{12} \mathrm{C}$ ions over the mask. Residues calculated from a linear fit to the data are presented in fig. 5. Data from the two outermost slits positioned at a distance of only $5 \mathrm{~mm}$ from the edges of the counter were not included in the fit, since at these places the delay-line readout system shows systematic errors (section 3.1). Also the electrical field in the ionization chamber is not very uniform near the edges of the counter. The maximum residue for the charge-division readout is $0.5 \mathrm{~mm}(0.2 \%$ of the total length), for the delay-line readout it is $1.0 \mathrm{~mm}(0.4 \%)$.

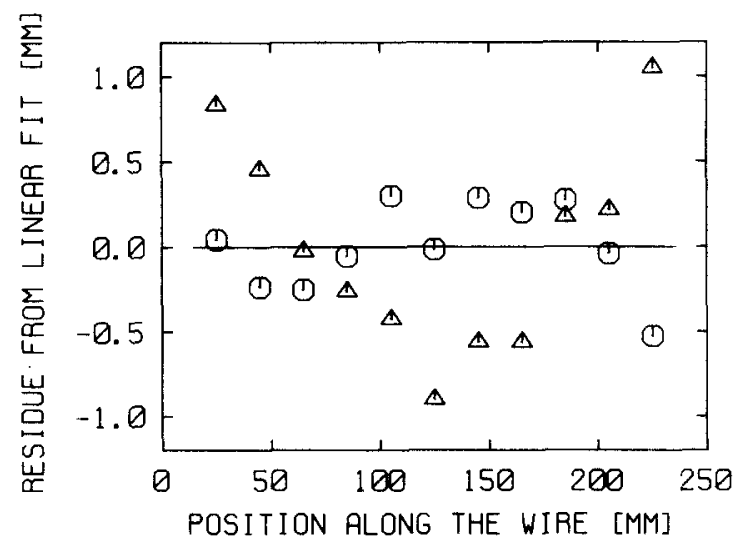

Fig. 5. The difference between the measured positions and a linear fit to the data for 11 positions covering $200 \mathrm{~mm}$ of the detector. The triangles give the residues for the delay-line readout, the circles those for the charge-division readout.

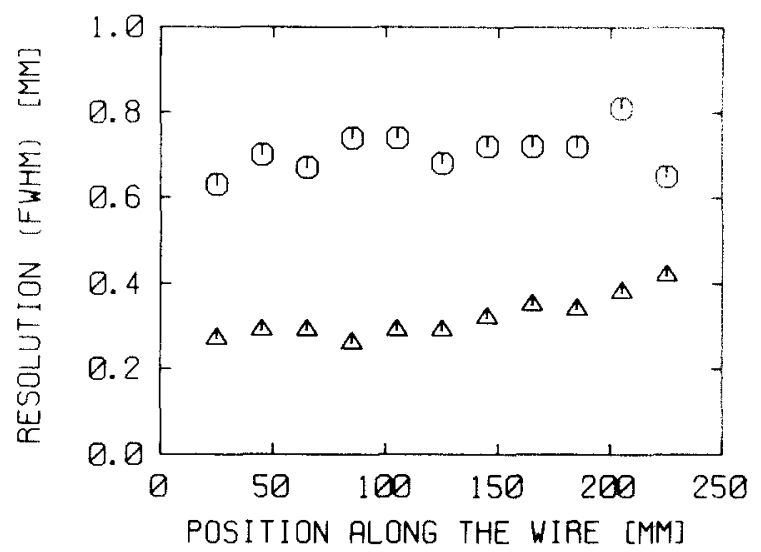

Fig. 6. The measured resolutions (fwhm) from delay-line (triangles) and charge-division readout (circles) for 11 positions covering $200 \mathrm{~mm}$ of the detector.

From fig. 5 it can be seen that the residues from the delay-line data show a systematic behaviour. Fitted with a quadratic curve the maximum residue for the delay-line decreases to $0.3 \mathrm{~mm}$ while the residues then show a random behaviour.

Fig. 6 shows the full width at half-maximum calculated from the data for the 11 slits. The delay-line resolution is found to be $0.3 \mathrm{~mm}$ which can be explained by the measured electronic resolution of 0.20 $\mathrm{mm}$ and a source width of $0.22 \mathrm{~mm}$, consistent with the slit width of $0.2 \mathrm{~mm}$. Angular straggling in entrance foil and gas is of minor importance under our experimental conditions. The full width at half-maximum increases somewhat near the edge of the counter, a geometrical effect which stems from the fact that for larger positions the distance between the focal plane and the mask decreases, which increases the source width. The mean fwhm for the charge-division readout is $0.7 \mathrm{~mm}$. By connecting a pulser to the test input of the two preamplifiers the contribution to the fwhm due to the electronic noise was found to be $0.65 \mathrm{~mm}$. Together with the source width this fully explains the fwhm of the charge-division readout.

\section{Conclusions}

A delay-line position readout for use in a "Rochester" type detector was constructed and compared with the standard charge-division readout. The resolution of the delay-line readout $(0.3 \mathrm{~mm} \mathrm{fwhm})$ is a factor 2 better than the resolution of the charge-division readout $(0.7 \mathrm{~mm}$ fwhm). Using a quadratic gauge curve the correspondence between measured and actual position for the delay-line readout was better then $0.3 \mathrm{~mm}$. 
Implementation of this simple and rugged constructed position readout, combining a better performance with an easy way of operation, will be a valuable improvement of the existing heavy-ion detection system.

This work was performed as part of the research program of the "Stichting voor Fundamenteel Onderzoek der Materie" (FOM) with financial support from the "Nederlandse Organisatie voor Zuiver-Wetenschappelijk Onderzoek" (ZWO).

\section{References}

[1] J.L.C. Ford, Nucl. Instr. and Meth. 162 (1979) 277.

[2] D. Shapira, R.M. DeVries, H.W. Fullbright, J. Toke and M.R. Clover, Nucl. Instr. and Meth. 129 (1975) 123.
[3] J.R. Erskine, T.H. Braid and J.C. Stolzfus, Nucl. Instr. and Meth. 135 (1976) 67.

[4] Annual Report University of Rochester (1979) p. 174.

[5] R.G. Markham and R.G.H. Robertson, Nucl. Instr. and Meth. 129 (1975) 131.

[6] Data Delay Devices Inc., New Jersey 07011, USA.

[7] E.J. de Graaf, A.M.J. Paans, M.G. Woldring and W.J.J. Spijkervet, Nucl. Instr, and Meth. 166 (1979) 139.

[8] I. Endo, T. Kawamoto, Y. Mizuno, T. Ohsugi, T. Taniguchi and T. Takeshita, Nucl. Instr. and Meth. 188 (1981) 51. 